\title{
Cumulant Generating Function of Codeword Lengths in Variable-Length Lossy Compression Allowing Positive Excess Distortion Probability
}

\author{
Shota Saito and Toshiyasu Matsushima \\ Department of Pure and Applied Mathematics, Waseda University \\ 3-4-1 Okubo, Shinjuku-ku, Tokyo, 169-8555 JAPAN \\ E-mail:wa-shota0425@fuji.waseda.jp and toshimat@waseda.jp
}

\begin{abstract}
This paper considers the problem of variable-length lossy source coding. The performance criteria are the excess distortion probability and the cumulant generating function of codeword lengths. We derive a non-asymptotic fundamental limit of the cumulant generating function of codeword lengths allowing positive excess distortion probability. It is shown that the achievability and converse bounds are characterized by the Rényi entropy-based quantity. In the proof of the achievability result, the explicit code construction is provided. Further, we investigate an asymptotic single-letter characterization of the fundamental limit for a stationary memoryless source.
\end{abstract}

\section{INTRODUCTION}

The problem of variable-length source coding is one of the fundamental research topics in Shannon theory. For this problem, one of the criteria is the normalized cumulant generating function of codeword lengths. This criterion was first proposed by Campbell [1] as a proxy for the mean codeword length.

Several previous works investigated the fundamental limit of the normalized cumulant generating function of codeword lengths: e.g., [1] and [2] for the problem of variable-length lossless source coding; [9] for the problem of variable-length source coding allowing errors; [3] for the problem of variablelength lossy source coding.

The most relevant study to this paper is the work by Courtade and Verdú [3]. As described above, they considered the problem of variable-length lossy source coding. As a criterion of the distortion measure, they treated the excess distortion probability. Their object of study was the code whose excess distortion probability is zero at a given distortion level $D$. By using the D-tilted Rényi entropy, the study [3] derived the converse bound for the fundamental limit of the normalized cumulant generating function of codeword lengths.

This paper considers the problem of variable-length lossy source coding and treats the same criteria as in [3]. However, the primary differences are 1) we evaluate the code whose excess distortion probability may be positive, and 2) we derive both achievability and converse bounds by using a novel Rényi entropy-based quantity. To show the achievability results, we give an explicit code construction instead of using the random coding argument.

Section II formulates the problem setup. Section III describes the related work by Courtade and Verdú [3]. Sections IV and V show the main results in this paper. In Section IV, we first define a Rényi entropy-based quantity. Then, using this quantity, we show non-asymptotic upper and lower bounds of the fundamental limit. Section V investigates an asymptotic single-letter characterization of the fundamental limit for a stationary memoryless source. Proofs of main results are in Section VI. Section VII discusses the obtained results.

\section{Problem Formulation}

Let $\mathcal{X}$ be a source alphabet and $\mathcal{Y}$ be a reproduction alphabet, where both are finite sets. Let $X$ be a random variable taking a value in $\mathcal{X}$ and $x$ be a realization of $X$. The probability distribution of $X$ is denoted as $P_{X}$. A distortion measure $d$ is defined as $d: \mathcal{X} \times \mathcal{Y} \rightarrow[0,+\infty)$.

The pair of an encoder and a decoder $(f, g)$ is defined as follows. An encoder $f$ is defined as $f: \mathcal{X} \rightarrow\{0,1\}^{\star}$, where $\{0,1\}^{\star}$ denotes the set of all finite-length binary strings and the empty string $\lambda$, i.e., $\{0,1\}^{\star}=\{\lambda, 0,1,00, \ldots\}$. An encoder $f$ is possibly stochastic and produces a nonprefix code. For $x \in \mathcal{X}$, the codeword length of $f(x)$ is denoted as $\ell(f(x))$. A deterministic decoder $g$ is defined as $g:\{0,1\}^{\star} \rightarrow \mathcal{Y}$. Variable-length lossy source coding without the prefix condition is discussed as in, for example, [3] and [8]. Once we prove a result for a non-prefix code, we can easily derive a result for a prefix code. We shall discuss it in Section VII.

For a code $(f, g)$, we define the excess distortion probability and the normalized cumulant generating function of codeword lengths.

Definition 1: Given $D \geq 0$, the excess distortion probability is defined as $\mathbb{P}[d(X, g(f(X)))>D]$.

Definition 2: Given $t>0$, the normalized cumulant generating function of codeword lengths is defined as 1

$$
\frac{1}{t} \log \mathbb{E}\left[2^{t \ell(f(X))}\right] \text {. }
$$

Remark 1: The 1'Hôspital theorem yields

$$
\begin{aligned}
\lim _{t \rightarrow 0} \frac{1}{t} \log \mathbb{E}\left[2^{t \ell(f(X))}\right] & =\mathbb{E}[\ell(f(X))], \\
\lim _{t \rightarrow \infty} \frac{1}{t} \log \mathbb{E}\left[2^{t \ell(f(X))}\right] & =\max _{x \in \mathcal{X}} \ell(f(x)) .
\end{aligned}
$$

\footnotetext{
${ }^{1}$ All logarithms are of base 2 throughout this paper. Further, $\exp \{\cdot\}$ denotes $2^{(\cdot)}$ in this paper.
} 
Thus, the normalized cumulant generating function of codeword lengths contains the mean codeword length and the maximum codeword length as its special cases.

Using these criteria, we define a $(D, R, \epsilon, t)$ code.

Definition 3: Given $D, R \geq 0, \epsilon \in[0,1)$, and $t>0$, a code $(f, g)$ satisfying

$$
\begin{aligned}
\mathbb{P}[d(X, g(f(X)))>D] & \leq \epsilon, \\
\frac{1}{t} \log \mathbb{E}\left[2^{t \ell(f(X))}\right] & \leq R
\end{aligned}
$$

is called a $(D, R, \epsilon, t)$ code.

The fundamental limit that we investigate is

$$
R^{*}(D, \epsilon, t):=\inf \{R: \exists \text { a }(D, R, \epsilon, t) \text { code }\} .
$$

When we work on the setup of blocklength $n$, we formulate the problem as follows. Let $\mathcal{X}^{n}$ and $\mathcal{Y}^{n}$ be the $n$-th Cartesian product of $\mathcal{X}$ and $\mathcal{Y}$, respectively. Let $X^{n}$ be a random variable taking a value in $\mathcal{X}^{n}$ and $x^{n}$ be a realization of $X^{n}$. The probability distribution of $X^{n}$ is denoted as $P_{X^{n}}$. A distortion measure $d_{n}$ is defined as $d_{n}: \mathcal{X}^{n} \times \mathcal{Y}^{n} \rightarrow[0,+\infty)$. An encoder $f_{n}: \mathcal{X}^{n} \rightarrow\{0,1\}^{\star}$ is possibly stochastic and produces a non-prefix code. A decoder $g_{n}:\{0,1\}^{\star} \rightarrow \mathcal{Y}^{n}$ is deterministic.

We define an $(n, D, R, \epsilon, t)$ code as follows.

Definition 4: Given $n \in \mathbb{N}, D, R \geq 0, \epsilon \in[0,1)$, and $t>0$, a code $\left(f_{n}, g_{n}\right)$ satisfying

$$
\begin{aligned}
\mathbb{P}\left[\frac{1}{n} d_{n}\left(X^{n}, g_{n}\left(f_{n}\left(X^{n}\right)\right)\right)>D\right] & \leq \epsilon, \\
\frac{1}{n t} \log \mathbb{E}\left[2^{t \ell\left(f_{n}\left(X^{n}\right)\right)}\right] & \leq R
\end{aligned}
$$

is called an $(n, D, R, \epsilon, t)$ code.

The fundamental limit is

$$
R^{*}(n, D, \epsilon, t):=\inf \{R: \exists \text { an }(n, D, R, \epsilon, t) \text { code }\}
$$

\section{Previous Study}

Courtade and Verdú [3] considered the same problem setting with the restriction that the code $(f, g)$ satisfies $\mathbb{P}[d(X, g(f(X)))>D]=0$ (i.e., $\epsilon=0$ in (4)). One of the main results in [3] is the converse bound on $R^{*}(D, 0, t)$. Before describing the result, we first introduce the $D$-tilted information [7] and D-tilted Rényi entropy [3].

Let $R(D)$ be the rate-distortion function, i.e.,

$$
R(D)=\min _{\substack{P_{Y \mid X}: \\ \mathbb{E}[d(X, Y)] \leq D}} I(X ; Y),
$$

where $I(X ; Y)$ denotes the mutual information between random variables $X$ and $Y$, and $P_{Y \mid X}$ denotes a conditional probability distribution of $Y$ given $X$. Assume that the minimum in the rate-distortion function $R(D)$ is achieved by $P_{Y \mid X}^{\star}$. Further, let $Y^{\star}$ be a random variable taking a value in $\mathcal{Y}$ and whose distribution $P_{Y \star}$ is the marginal of $P_{Y}^{\star} \mid X P_{X}$. Then, the $D$-tilted information of $x \in \mathcal{X}$ is defined as

$$
\jmath_{X}(x, D)=\log \frac{1}{\mathbb{E}\left[\exp \left\{\lambda^{\star} D-\lambda^{\star} d\left(x, Y^{\star}\right)\right\}\right]},
$$

where the expectation is with respect to $P_{Y^{\star}}$ and $\lambda^{\star}:=$ $-R^{\prime}(D)$. Further, the $D$-tilted Rényi entropy of order $\alpha \in$ $(0,1) \cup(1, \infty)$ is defined as [3]

$$
H_{\alpha}(X, D)=\frac{1}{1-\alpha} \log \mathbb{E}\left[2^{(1-\alpha)_{X X}(X, D)}\right] .
$$

The next theorem characterizes the converse bound on $R^{*}(D, 0, t)$ by the $D$-tilted Rényi entropy.

Theorem 1 ( [3]): For any $D \geq 0$ and $t>0$,

$$
R^{*}(D, 0, t) \geq H_{\frac{1}{1+t}}(X, D)-\log \log (1+\min \{|\mathcal{X}|,|\mathcal{Y}|\}),
$$

where $|\mathcal{X}|$ and $|\mathcal{Y}|$ represent the cardinality of $\mathcal{X}$ and $\mathcal{Y}$, respectively.

Remark 2: The previous study [3] investigated the case where the excess distortion probability is zero (i.e., $\epsilon=0$ in (4)). Further, they only showed the converse result. On the other hand, our study deals with positive excess distortion probability as in (4). Moreover, our study investigates both achievability and converse bounds.

\section{NON-ASYMPTOTIC ANALYSIS}

\section{A. Preliminary: Rényi Entropy-Based Quantity}

For $\alpha \in(0,1) \cup(1, \infty)$, the Rényi entropy is defined as [12]

$$
H_{\alpha}(X)=\frac{1}{1-\alpha} \log \sum_{x \in \mathcal{X}}\left[P_{X}(x)\right]^{\alpha} .
$$

One of the useful properties of the Rényi entropy is Schur concavity. This property is used in the proof of the achievability result in our main theorem. To state the definition of a Schur concave function, we first review the notion of majorization.

Definition 5: Let $\mathbb{R}_{+}$be the set of non-negative real numbers and $\mathbb{R}_{+}^{m}$ be the $m$-th Cartesian product of $\mathbb{R}_{+}$, where $m$ is a positive integer. Suppose that $\mathbf{x}=\left(x_{1}, \ldots, x_{m}\right) \in \mathbb{R}_{+}^{m}$ and $\mathbf{y}=\left(y_{1}, \ldots, y_{m}\right) \in \mathbb{R}_{+}^{m}$ satisfy $x_{i} \geq x_{i+1}, y_{i} \geq y_{i+1}$ $(i=1,2, \ldots, m-1)$. If $\mathbf{x} \in \mathbb{R}_{+}^{m}$ and $\mathbf{y} \in \mathbb{R}_{+}^{m}$ satisfy, for $k=$ $1, \ldots, m-1, \sum_{i=1}^{k} x_{i} \leq \sum_{i=1}^{k} y_{i}$ and $\sum_{i=1}^{m} x_{i}=\sum_{i=1}^{m} y_{i}$, then we say that $\mathbf{y}$ majorizes $\mathbf{x}$ (it is denoted as $\mathbf{x} \prec \mathbf{y}$ in this paper).

Schur concave functions are defined as follows.

Definition 6: We say that a function $h(\cdot): \mathbb{R}_{+}^{m} \rightarrow \mathbb{R}$ is a Schur concave function if $h(\mathbf{y}) \leq h(\mathbf{x})$ for any $\mathbf{x}, \mathbf{y} \in \mathbb{R}_{+}^{m}$ satisfying $\mathbf{x} \prec \mathbf{y}$.

For any $\alpha \in(0,1) \cup(1, \infty)$, the Rényi entropy $H_{\alpha}(X)$ is a Schur concave function (see, e.g., [10]).

Next, we introduce a new quantity based on the Rényi entropy. This quantity plays an important role in producing our main results.

${ }^{2}$ Kostina and Verdú [7] named this quantity the $D$-tilted information. However, this quantity was used in earlier work by, e.g., Kontoyiannis [5]. 
Definition 7: Given $D \geq 0, \epsilon \in[0,1)$, and $\alpha \in(0,1) \cup$ $(1, \infty), G_{\alpha}^{D, \epsilon}(X)$ is defined as

$$
G_{\alpha}^{D, \epsilon}(X)=\min _{\substack{P_{Y \mid X}: \\ \mathbb{P}[d(X, Y)>D] \leq \epsilon}} H_{\alpha}(Y) .
$$

Remark 3: For a given $D \geq 0$ and $\epsilon \in[0,1)$, suppose that

$$
\mathbb{P}\left[\inf _{y \in \mathcal{Y}} d(X, y)>D\right]>\epsilon .
$$

Then, there are no codes whose excess distortion probability is less than or equal to $\epsilon$. Conversely, if such codes do not exist for given $D$ and $\epsilon$, (16) holds. In this case, we define $R^{*}(D, \epsilon, t)=+\infty$. Further, if 16 holds, we also define $G_{\alpha}^{D, \epsilon}(X)=+\infty$ because there is no conditional probability distribution $P_{Y \mid X}$ on $\mathcal{Y}$ satisfying $\mathbb{P}[d(X, Y)>D] \leq \epsilon$.

\section{B. Non-Asymptotic Coding Theorem}

The next lemma shows the achievability result on $R$ of a $(D, R, \epsilon, t)$ code.

Lemma 1: For any $D \geq 0, \epsilon \in[0,1)$, and $t>0$, there exists a $(D, R, \epsilon, t)$ code such that

$$
R=G_{\frac{1}{1+t}}^{D, \epsilon}(X) .
$$

Proof: See Section VI-A.

Remark 4: The random coding argument is not used to prove the achievability result. Instead, an explicit code construction is given. This is similar to Feinstein's cookie-cutting argument [4].

The next lemma shows the converse bound on $R$ of a $(D, R, \epsilon, t)$ code.

Lemma 2: For any $D \geq 0, \epsilon \in[0,1)$, and $t>0$, any $(D, R, \epsilon, t)$ code satisfies

$$
R \geq G_{\frac{1}{1+t}}^{D, \epsilon}(X)-\log \log (1+\min \{|\mathcal{X}|,|\mathcal{Y}|\}) .
$$

Proof: See Section VI-B.

Combining Lemmas 1 and 2, we can immediately obtain the following result on $R^{*}(D, \epsilon, t)$.

Theorem 2: For any $D \geq 0, \epsilon \in[0,1)$, and $t>0$,

$$
\begin{aligned}
& G_{\frac{1}{1+t}}^{D, \epsilon}(X)-\log \log (1+\min \{|\mathcal{X}|,|\mathcal{Y}|\}) \leq R^{*}(D, \epsilon, t) \\
& \leq G_{\frac{1}{1+t}}^{D, \epsilon}(X) .
\end{aligned}
$$

The same discussion which is used to prove Theorem 2 establishes the next result on $R^{*}(n, D, \epsilon, t)$.

Theorem 3: For any $n \in \mathbb{N}, D \geq 0, \epsilon \in[0,1)$, and $t>0$,

$$
\begin{aligned}
& \frac{1}{n} G_{\frac{1}{1+t}}^{D, \epsilon}\left(X^{n}\right)-\frac{1}{n} \log \log \left(1+\min \left\{\left|\mathcal{X}^{n}\right|,\left|\mathcal{Y}^{n}\right|\right\}\right) \\
& \leq R^{*}(n, D, \epsilon, t) \leq \frac{1}{n} G_{\frac{1}{1+t}}^{D, \epsilon}\left(X^{n}\right),
\end{aligned}
$$

where $G_{\frac{1}{1+t}}^{D, \epsilon}\left(X^{n}\right)$ is defined as

$$
G_{\frac{1}{1+t}}^{D, \epsilon}\left(X^{n}\right)=\min _{\substack{P_{Y^{n} \mid X^{n}:}: \\ \mathbb{P}\left[d_{n}(X, Y)>n D\right] \leq \epsilon}} H_{\frac{1}{1+t}}\left(Y^{n}\right) .
$$

\section{Asymptotic AnAlysis FOR a StATIONARY MEMORYLESS SOURCE}

This section investigates the general formula (20) when a stationary memoryless source is assumed. Especially, we consider the special case $t \downarrow 0$ and drive a single-letter characterization of the fundamental limit $R^{*}(n, D, \epsilon, 0):=$ $\lim _{t \downarrow 0} R^{*}(n, D, \epsilon, t)$.

First, two quantities are defined. As we show in Section VI-C they are closely related to the quantity $\lim _{\alpha \uparrow 1} G_{\alpha}^{D, \epsilon}\left(X^{n}\right)$.

Definition 8: Given $D \geq 0$ and $\epsilon \in[0,1)$, the $(D, \epsilon)$-entropy $H_{D, \epsilon}\left(X^{n}\right)$ is defined as [11]

$$
H_{D, \epsilon}\left(X^{n}\right)=\min _{\substack{\varphi: \mathcal{X}^{n} \rightarrow \mathcal{Y}^{n}: \\ \mathbb{P}\left[d_{n}\left(X^{n}, \varphi\left(X^{n}\right)\right)>n D\right] \leq \epsilon}} H\left(\varphi\left(X^{n}\right)\right),
$$

where $H(\cdot)$ denotes the Shannon entropy.

Definition 9: Given $D \geq 0$ and $\epsilon \in[0,1)$, the quantity $R_{D, \epsilon}\left(X^{n}\right)$ is defined as

$$
R_{D, \epsilon}\left(X^{n}\right)=\underset{\substack{P_{Y^{n} \mid X}^{n}: \\ \mathbb{P}\left[d_{n}\left(X^{n}, Y^{n}\right)>n D\right] \leq \epsilon}}{\min } I\left(X^{n} ; Y^{n}\right) .
$$

Kostina et al. [8] showed the next asymptotic result on $H_{D, \epsilon}\left(X^{n}\right)$ and $R_{D, \epsilon}\left(X^{n}\right)$.

Theorem 4 ( [8]): We impose the next assumptions:

1) For $\left(x^{n}, y^{n}\right) \in \mathcal{X}^{n} \times \mathcal{Y}^{n}$, the distortion measure $d_{n}\left(x^{n}, y^{n}\right)$ satisfies $d_{n}\left(x^{n}, y^{n}\right)=\sum_{i=1}^{n} d\left(x_{i}, y_{i}\right)$.

2) The distortion level $D$ satisfies $D \in\left(D_{\min }, D_{\max }\right)$, where $D_{\min }:=\inf \{D: R(D)<\infty\}$ and $D_{\max }:=$ $\inf _{y \in \mathcal{Y}} \mathbb{E}[d(X, y)]$.

3) The minimum in the rate-distortion function $R(D)$ is achieved by $P_{Y \mid X}^{\star}$.

4) $\mathbb{E}\left[d^{12}\left(X, Y^{\star}\right)\right]<\infty$, where the expectation is with respect to $P_{X} \times P_{Y^{\star}}$.

Under a stationary memoryless source and the assumptions 1) - 4), we have, for any $\epsilon \in[0,1)$,

$$
\begin{aligned}
& H_{D, \epsilon}\left(X^{n}\right)=R_{D, \epsilon}\left(X^{n}\right) \\
& =(1-\epsilon) n R(D)-\sqrt{\frac{n V(D)}{2 \pi}} e^{-\frac{\left(Q^{-1}(\epsilon)\right)^{2}}{2}}+O(\log n),
\end{aligned}
$$

where $V(D)$ is the rate-dispersion function [7] which is defined as the variance of the $D$-tilted information, i.e., $V(D):=\operatorname{Var}\left[\jmath_{X}(X, D)\right]$ and $Q^{-1}(z)$ denotes the inverse function of $Q(z)=\int_{z}^{\infty}(1 / \sqrt{2 \pi}) \exp \left(-t^{2} / 2\right) d t$ for $z \in \mathbb{R}$.

Combination of Theorems 3 and 4 leads to the next singleletter characterization on $R^{*}(n, D, \epsilon, 0)$.

Theorem 5: Under a stationary memoryless source and the assumptions 1) -4) in Theorem 4 , we have, for any $\epsilon \in[0,1)$,

$$
\begin{aligned}
& R^{*}(n, D, \epsilon, 0) \\
& =(1-\epsilon) R(D)-\sqrt{\frac{V(D)}{2 \pi n}} e^{-\frac{\left(Q^{-1}(\epsilon)\right)^{2}}{2}}+O\left(\frac{\log n}{n}\right) .
\end{aligned}
$$

\section{Proof: See Section VI-C}

Remark 5: In view of Remark 1, we observe that $R^{*}(n, D, \epsilon, 0)$ represents the fundamental limit of the mean 
codeword length. This quantity was investigated by [8], and our result (25) coincides with the result in [8].

\section{Proof of Main Results}

\section{A. Proof of Lemma 1}

First, some notations are defined before showing the construction of the encoder and the decoder.

- For any $y \in \mathcal{Y}$ and $D \geq 0, \mathcal{B}_{D}(y)$ is defined as

$$
\mathcal{B}_{D}(y)=\{x \in \mathcal{X}: d(x, y) \leq D\} .
$$

- We define $y_{i}(i=1,2, \cdots)$ by the following procedure. Let $y_{1}$ be defined as

$$
y_{1}=\underset{y \in \mathcal{Y}}{\arg \max } \mathbb{P}\left[X \in \mathcal{B}_{D}(y)\right] .
$$

For $i=2,3, \cdots$, let $y_{i}$ be defined as

$$
y_{i}=\underset{y \in \mathcal{Y}}{\arg \max } \mathbb{P}\left[X \in \mathcal{B}_{D}(y) \backslash \bigcup_{j=1}^{i-1} \mathcal{B}_{D}\left(y_{j}\right)\right] \text {. }
$$

- For $i=1,2, \ldots$, we define $\mathcal{A}_{D}\left(y_{i}\right)$ by

$$
\begin{aligned}
& \mathcal{A}_{D}\left(y_{1}\right)=\mathcal{B}_{D}\left(y_{1}\right), \\
& \mathcal{A}_{D}\left(y_{i}\right)=\mathcal{B}_{D}\left(y_{i}\right) \backslash \bigcup_{j=1}^{i-1} \mathcal{B}_{D}\left(y_{j}\right) \quad(\forall i \geq 2) .
\end{aligned}
$$

From the definition, we have

$$
\begin{aligned}
& \bigcup_{j=1}^{i} \mathcal{A}_{D}\left(y_{j}\right)=\bigcup_{j=1}^{i} \mathcal{B}_{D}\left(y_{j}\right) \quad(i \geq 1), \\
& \mathcal{A}_{D}\left(y_{i}\right) \cap \mathcal{A}_{D}\left(y_{j}\right)=\emptyset \quad(\forall i \neq j), \\
& \mathbb{P}\left[X \in \mathcal{A}_{D}\left(y_{1}\right)\right] \geq \mathbb{P}\left[X \in \mathcal{A}_{D}\left(y_{2}\right)\right] \geq \cdots .
\end{aligned}
$$

- Given $\epsilon \in[0,1)$, let $k^{*} \geq 1$ be the integer satisfying

$$
\begin{gathered}
\sum_{i=1}^{k^{*}-1} \mathbb{P}\left[X \in \mathcal{A}_{D}\left(y_{i}\right)\right]<1-\epsilon, \\
\sum_{i=1}^{k^{*}} \mathbb{P}\left[X \in \mathcal{A}_{D}\left(y_{i}\right)\right] \geq 1-\epsilon .
\end{gathered}
$$

- Let $\alpha$ and $\beta$ be defined as

$$
\begin{aligned}
& \alpha=\sum_{i=1}^{k^{*}-1} \mathbb{P}\left[X \in \mathcal{A}_{D}\left(y_{i}\right)\right], \\
& \beta=1-\epsilon-\alpha .
\end{aligned}
$$

- Let $w_{i}$ be the $i$-th binary string in $\{0,1\}^{\star}$ in the increasing order of the length and ties are arbitrarily broken. For example, $w_{1}=\lambda, w_{2}=0, w_{3}=1, w_{4}=00, w_{5}=01$, etc.

Using these notations, we construct the following encoder $\hat{f}: \mathcal{X} \rightarrow\{0,1\}^{\star}$ and decoder $\hat{g}:\{0,1\}^{\star} \rightarrow \mathcal{Y}$.

\section{[Encoder]}

1) For $x \in \mathcal{A}_{D}\left(y_{i}\right)\left(i=1, \ldots, k^{*}-1\right)$, set $\hat{f}(x)=w_{i}$.
2) For $x \in \mathcal{A}_{D}\left(y_{k^{*}}\right)$, se 3

$$
\hat{f}(x)= \begin{cases}w_{k^{*}} & \text { with prob. } \frac{\beta}{\mathbb{P}\left[X \in \mathcal{A}_{D}\left(y_{k^{*}}\right)\right]}, \\ w_{1} & \text { with prob. } 1-\frac{\beta}{\mathbb{P}\left[X \in \mathcal{A}_{D}\left(y_{k^{*}}\right)\right]} .\end{cases}
$$

3) For $x \notin \bigcup_{i=1}^{k^{*}} \mathcal{A}_{D}\left(y_{i}\right)$, set $\hat{f}(x)=w_{1}$.

[Decoder] Set $\hat{g}\left(w_{i}\right)=y_{i}\left(i=1, \ldots, k^{*}\right)$.

Now, we evaluate the excess distortion probability. We have $d(x, \hat{g}(\hat{f}(x))) \leq D$ for $x \in \mathcal{A}_{D}\left(y_{i}\right)\left(i=1, \ldots, k^{*}-1\right)$ since $\hat{g}(\hat{f}(x))=y_{i}$. Furthermore, we have $d(x, \hat{g}(\hat{f}(x))) \leq D$ with probability $\beta / \mathbb{P}\left[X \in \mathcal{A}_{D}\left(y_{k^{*}}\right)\right]$ for $x \in \mathcal{A}_{D}\left(y_{k^{*}}\right)$. Thus,

$$
\begin{aligned}
& \mathbb{P}[d(X, \hat{g}(\hat{f}(X))) \leq D] \\
& =\sum_{i=1}^{k^{*}-1} \mathbb{P}\left[X \in \mathcal{A}_{D}\left(y_{i}\right)\right]+\mathbb{P}\left[\hat{f}(X)=w_{k^{*}}, X \in \mathcal{A}_{D}\left(y_{k^{*}}\right)\right] \\
& =\alpha+\beta=1-\epsilon .
\end{aligned}
$$

Therefore, we have $\mathbb{P}[d(X, \hat{g}(\hat{f}(X)))>D]=\epsilon$.

Next, we evaluate the normalized cumulant generating function of codeword lengths for the code $(\hat{f}, \hat{g})$. To this end, we denote by $\hat{Y}:=\hat{g}(\hat{f}(X))$ and show the next lemma.

Lemma 3: For any $t>0$ and $i \in\left\{1,2, \ldots, k^{*}\right\}$, we have

$$
2^{t \ell\left(\hat{g}^{-1}\left(y_{i}\right)\right)} \leq i^{t} \leq\left[\sum_{j=1}^{k^{*}}\left(\frac{P_{\hat{Y}}\left(y_{j}\right)}{P_{\hat{Y}}\left(y_{i}\right)}\right)^{\frac{1}{1+t}}\right]^{t},
$$

where $\hat{g}^{-1}$ denotes the inverse function ${ }^{4}$ of $\hat{g}$.

Proof: First, we show the left inequality of (41). The construction of the code gives

$$
\ell\left(\hat{g}^{-1}\left(y_{i}\right)\right) \leq \log i
$$

for any $i \in\left\{1,2, \ldots, k^{*}\right\}$. This inequality yields

$$
2^{t \ell\left(\hat{g}^{-1}\left(y_{i}\right)\right)} \leq 2^{t \log i}=i^{t},
$$

which is the left inequality of (41).

Next, we show the right inequality of 41]. The code construction gives the next inequality on the distribution of $\hat{Y}$ :

$$
P_{\hat{Y}}\left(y_{1}\right) \geq P_{\hat{Y}}\left(y_{2}\right) \geq \ldots \geq P_{\hat{Y}}\left(y_{k^{*}}\right) \text {. }
$$

Thus, for any $i \in\left\{1,2, \ldots, k^{*}\right\}$, it follows that

$$
\begin{aligned}
& \left(\frac{P_{\hat{Y}}\left(y_{1}\right)}{P_{\hat{Y}}\left(y_{i}\right)}\right)^{\frac{1}{1+t}} \geq 1, \quad\left(\frac{P_{\hat{Y}}\left(y_{2}\right)}{P_{\hat{Y}}\left(y_{i}\right)}\right)^{\frac{1}{1+t}} \geq 1, \\
& \ldots,\left(\frac{P_{\hat{Y}}\left(y_{i-1}\right)}{P_{\hat{Y}}\left(y_{i}\right)}\right)^{\frac{1}{1+t}} \geq 1, \quad\left(\frac{P_{\hat{Y}}\left(y_{i}\right)}{P_{\hat{Y}}\left(y_{i}\right)}\right)^{\frac{1}{1+t}}=1 .
\end{aligned}
$$

${ }^{3}$ Note that we have $\mathbb{P}\left[X \in \mathcal{A}_{D}\left(y_{k^{*}}\right)\right] \geq \beta$ from 35.

${ }^{4}$ From the construction of $\hat{g}$, we can define its inverse function. 
Hence, for any $i \in\left\{1,2, \ldots, k^{*}\right\}$, we have

$$
\begin{aligned}
& i=\underbrace{1+1+\cdots+1}_{i} \\
& \stackrel{(a)}{\leq}\left(\frac{P_{\hat{Y}}\left(y_{1}\right)}{P_{\hat{Y}}\left(y_{i}\right)}\right)^{\frac{1}{1+t}}+\left(\frac{P_{\hat{Y}}\left(y_{2}\right)}{P_{\hat{Y}}\left(y_{i}\right)}\right)^{\frac{1}{1+t}}+\cdots+\left(\frac{P_{\hat{Y}}\left(y_{i}\right)}{P_{\hat{Y}}\left(y_{i}\right)}\right)^{\frac{1}{1+t}} \\
& \stackrel{(b)}{\leq} \sum_{j=1}^{k^{*}}\left(\frac{P_{\hat{Y}}\left(y_{j}\right)}{P_{\hat{Y}}\left(y_{i}\right)}\right)^{\frac{1}{1+t}}
\end{aligned}
$$

where $(a)$ follows from (45) and $(b)$ is due to

$$
\frac{P_{\hat{Y}}\left(y_{j}\right)}{P_{\hat{Y}}\left(y_{i}\right)} \geq 0 \quad\left(\forall i, j \in\left\{1,2, \ldots, k^{*}\right\}\right) .
$$

The inequality (48) yields the right inequality of (41).

Using Lemma 3, we have

$$
\begin{aligned}
\mathbb{E}\left[2^{t \ell\left(\hat{g}^{-1}(\hat{Y})\right)}\right] & =\sum_{i=1}^{k^{*}} P_{\hat{Y}}\left(y_{i}\right) 2^{t \ell\left(\hat{g}^{-1}\left(y_{i}\right)\right)} \\
& \leq \sum_{i=1}^{k^{*}} P_{\hat{Y}}\left(y_{i}\right)\left[\sum_{j=1}^{k^{*}}\left(\frac{P_{\hat{Y}}\left(y_{j}\right)}{P_{\hat{Y}}\left(y_{i}\right)}\right)^{\frac{1}{1+t}}\right]^{t} \\
& =\left(\sum_{j=1}^{k^{*}}\left[P_{\hat{Y}}\left(y_{j}\right)\right]^{\frac{1}{1+t}}\right)^{1+t} .
\end{aligned}
$$

Thus, taking logarithm of both sides of (52) and dividing by $t>0$, we have

$$
\begin{aligned}
\frac{1}{t} \log \mathbb{E}\left[2^{t \ell\left(\hat{g}^{-1}(\hat{Y})\right)}\right] & \leq \frac{1+t}{t} \log \sum_{j=1}^{k^{*}}\left[P_{\hat{Y}}\left(y_{j}\right)\right]^{\frac{1}{1+t}} \\
& =H_{\frac{1}{1+t}}(\hat{Y}) .
\end{aligned}
$$

Finally, we evaluate the left and right-hand sides of (54). The left-hand side of (54) is evaluated as

$$
\mathbb{E}\left[2^{t \ell\left(\hat{g}^{-1}(\hat{Y})\right)}\right]=\mathbb{E}\left[2^{t \ell(\hat{f}(X))}\right] .
$$

Indeed, this is verified as follows:

$$
\begin{aligned}
\mathbb{E}\left[2^{t \ell(\hat{f}(X))}\right] & =\sum_{x \in \mathcal{X}} P_{X}(x) 2^{t \ell(\hat{f}(x))} \\
& =\sum_{i=1}^{k^{*}} \mathbb{P}\left[\hat{f}(X)=w_{i}\right] 2^{t \ell\left(w_{i}\right)} \\
& =\sum_{i=1}^{k^{*}} \mathbb{P}\left[\hat{g}^{-1}(\hat{Y})=w_{i}\right] 2^{t \ell\left(w_{i}\right)} \\
& =\sum_{i=1}^{k^{*}} P_{\hat{Y}}\left(y_{i}\right) 2^{t \ell\left(\hat{g}^{-1}\left(y_{i}\right)\right)} \\
& =\mathbb{E}\left[2^{t \ell\left(\hat{g}^{-1}(\hat{Y})\right)}\right] .
\end{aligned}
$$

On the other hand, the right-hand side of (54) is evaluated as

$$
H_{\frac{1}{1+t}}(\hat{Y})=G_{\frac{1}{1+t}}^{D, \epsilon}(X) .
$$

This is proved by combining the fact that the Rényi entropy is a Schur concave function and the next lemma shown in [13].

Lemma 4 ( [13] $)$ : The distribution $P_{\hat{Y}}$ majorizes any $P_{\tilde{Y}}$ induced by $P_{\tilde{Y} \mid X}$ satisfying $\mathbb{P}[d(X, \tilde{Y})>D] \leq \epsilon$.

Therefore, the combination of (54), (55), and (61) gives

$$
\frac{1}{t} \log \mathbb{E}\left[2^{t \ell(\hat{f}(X))}\right] \leq G_{\frac{1}{1+t}}^{D, \epsilon}(X),
$$

which completes the proof of Lemma 1.

\section{B. Proof of Lemma 2}

Fix a $(D, R, \epsilon, t)$ code $(f, g)$ arbitrarily and we denote by $\bar{Y}:=g(f(X))$. Further, without loss of generality, we assume that the decoder $g$ is an injective mapping ${ }^{5}$. Then, the definition of a $(D, R, \epsilon, t)$ code gives

$$
\begin{aligned}
\mathbb{P}[d(X, \bar{Y})>D] & \leq \epsilon, \\
\frac{1}{t} \log \mathbb{E}\left[2^{t \ell(f(X))}\right] & \leq R,
\end{aligned}
$$

and the assumption that $g$ is an injective mapping yields the next inequality [2]:

$$
\sum_{y \in \overline{\mathcal{Y}}} 2^{-\ell\left(g^{-1}(y)\right)} \leq \log (1+\min \{|\mathcal{X}|,|\mathcal{Y}|\})
$$

where $\overline{\mathcal{Y}}:=\{g(f(x)): x \in \mathcal{X}\} \subset \mathcal{Y}$.

The key lemma in the proof of the converse result is as follows.

Lemma 5: For any $t>0$, we have

$\frac{1}{t} \log \mathbb{E}\left[2^{t \ell\left(g^{-1}(\bar{Y})\right)}\right] \geq H_{\frac{1}{1+t}}(\bar{Y})-\log \sum_{y \in \overline{\mathcal{Y}}} 2^{-\ell\left(g^{-1}(y)\right)}$.

Proof: For each $y \in \overline{\mathcal{Y}}$,

$$
\begin{aligned}
& \alpha(y):=\left[2^{\ell\left(g^{-1}(y)\right)}\right]^{-\frac{t}{1+t}} \\
& \beta(y):=\left[P_{\bar{Y}}(y)\right]^{\frac{1}{1+t}}\left[2^{\ell\left(g^{-1}(y)\right)}\right]^{\frac{t}{1+t}} .
\end{aligned}
$$

Then, Hölder's inequality gives

$$
\sum_{y \in \overline{\mathcal{Y}}} \alpha(y) \beta(y) \leq\left(\sum_{y \in \overline{\mathcal{Y}}}[\alpha(y)]^{\frac{1+t}{t}}\right)^{\frac{t}{1+t}}\left(\sum_{y \in \overline{\mathcal{Y}}}[\beta(y)]^{1+t}\right)^{\frac{1}{1+t}} .
$$

Taking logarithm of both sides of 69) and substituting 67 and (68) for 69), we obtain

$$
\begin{aligned}
& \frac{1+t}{t} \log \sum_{y \in \overline{\mathcal{Y}}}\left[P_{\bar{Y}}(y)\right]^{\frac{1}{1+t}} \\
& \leq \log \sum_{y \in \overline{\mathcal{Y}}} 2^{-\ell\left(g^{-1}(y)\right)}+\frac{1}{t} \log \mathbb{E}\left[2^{\left.t \ell\left(g^{-1}(\bar{Y})\right)\right)}\right] .
\end{aligned}
$$

\footnotetext{
${ }^{5}$ Note that it is sufficient to consider the case where the decoder $g$ is an
} injective mapping in the proof of the converse part (see, e.g., [3]). 
Further, noticing that the left hand side of (70) is

$$
\frac{1+t}{t} \log \sum_{y \in \overline{\mathcal{Y}}}\left[P_{\bar{Y}}(y)\right]^{\frac{1}{1+t}}=H_{\frac{1}{1+t}}(\bar{Y}),
$$

we obtain the desired result (66).

Combination of (65), (66), and $\mathbb{E}\left[2^{t \ell(f(X))}\right]=$ $\mathbb{E}\left[2^{t \ell\left(g^{-1}(\bar{Y})\right)}\right]$ yields

$$
\begin{aligned}
& \frac{1}{t} \log \mathbb{E}\left[2^{t \ell(f(X))}\right] \\
& \left.\quad \geq H_{\frac{1}{1+t}}(\bar{Y})-\log \log (1+\min \{|\mathcal{X}|,|\mathcal{Y}|\})\right] .
\end{aligned}
$$

Finally, from (63) and 64), we have (18).

\section{Proof of Theorem 5}

We denote by $G_{1}^{D, \epsilon}\left(X^{n}\right):=\lim _{\alpha \uparrow 1} G_{\alpha}^{D, \epsilon}\left(X^{n}\right)$ and $\hat{Y}^{n}:=$ $\hat{g}_{n}\left(\hat{f}_{n}\left(X^{n}\right)\right)$, where $\left(\hat{f}_{n}, \hat{g}_{n}\right)$ is the code as constructed in the proof of Lemma 1. Then, we have

$$
\begin{gathered}
G_{1}^{D, \epsilon}\left(X^{n}\right)=\lim _{\alpha \uparrow 1} G_{\alpha}^{D, \epsilon}\left(X^{n}\right) \stackrel{(a)}{=} \lim _{\alpha \uparrow 1} H_{\alpha}\left(\hat{Y}^{n}\right) \\
\stackrel{(b)}{=} H\left(\hat{Y}^{n}\right) \stackrel{(c)}{=} \underset{\substack{\left.P_{Y^{n} \mid X n}\right) \\
\mathbb{P}\left[d_{n}\left(X^{n}, Y^{n}\right)>n D\right] \leq \epsilon}}{\min _{n}} H\left(Y^{n}\right),
\end{gathered}
$$

where (a) follows from (61), (b) is due to the fact that the Rényi entropy approaches the Shannon entropy as $\alpha$ tends to 1, and (c) follows from Lemma 4 and the fact that the Shannon entropy is a Schur concave function (e.g., [10]).

Further, the definition of $H_{D, \epsilon}\left(X^{n}\right)$ gives

$$
\min _{\substack{\left.\left.P_{Y^{n} \mid X^{n}}: \\ X_{n}, Y^{n}\right)>n D\right] \leq \epsilon}} H\left(Y^{n}\right) \leq H_{D, \epsilon}\left(X^{n}\right) .
$$

Combination of (74) and (75) yields

$$
G_{1}^{D, \epsilon}\left(X^{n}\right) \leq H_{D, \epsilon}\left(X^{n}\right) .
$$

On the other hand, we have

$$
\begin{aligned}
& R_{D, \epsilon}\left(X^{n}\right)=\min _{\mathbb{P}\left[d_{n}\left(X_{Y^{n} \mid X^{n}}^{n}, Y^{n}\right)>n D\right] \leq \epsilon} I\left(X^{n} ; Y^{n}\right) \\
& \stackrel{(a)}{\leq} \min _{\substack{P_{Y^{n} \mid X^{n}}: \\
\mathbb{P}\left[d_{n}\left(X^{n}, Y^{n}\right)>n D\right] \leq \epsilon}} H\left(Y^{n}\right) \stackrel{(b)}{=} G_{1}^{D, \epsilon}\left(X^{n}\right),
\end{aligned}
$$

where (a) is due to the non-negativity of the conditional Shannon entropy and (b) follows from (74).

Thus, combination of (76) and (78) and application of Theorem 4 establish

$$
\begin{aligned}
& G_{1}^{D, \epsilon}\left(X^{n}\right) \\
& =(1-\epsilon) n R(D)-\sqrt{\frac{n V(D)}{2 \pi}} e^{-\frac{\left(Q^{-1}(\epsilon)\right)^{2}}{2}}+O(\log n) .
\end{aligned}
$$

Finally, letting $t \downarrow 0$ in Theorem 3, using (79), and noticing

$$
\frac{1}{n} \log \log \left(1+\min \left\{\left|\mathcal{X}^{n}\right|,\left|\mathcal{Y}^{n}\right|\right\}\right)=O\left(\frac{\log n}{n}\right),
$$

we obtain the desired result $(25)$.

\section{DISCUSSION}

\section{A. Theorem for a Deterministic Code}

So far, we have treated a stochastic code. If we deal with only a deterministic code, we have the next lemma instead of Lemma 1

Lemma 6: For any $D \geq 0, \epsilon \in[0,1)$, define $\gamma$ as $\gamma=$ $1-\sum_{i=1}^{k^{*}} \mathbb{P}\left[X \in \mathcal{A}_{D}\left(y_{i}\right)\right]$, where $\mathcal{A}_{D}\left(y_{i}\right)$ is defined as in (29) and (30) and $k^{*}$ is the integer satisfying (34) and (35). Then, for any $t>0$, there exists a deterministic $(D, R, \epsilon, t)$ code such that

$$
R=G_{\frac{1}{1+t}}^{D, \epsilon}(X)+\frac{(\epsilon-\gamma) \beta^{-\frac{t}{1+t}} \log e}{t \exp \left\{\frac{t}{1+t} G_{\frac{1}{1+t}}^{D, \epsilon}(X)\right\}},
$$

where $\beta$ is defined as in (37).

Proof: See Appendix A

Comparing Lemmas 1 and 6, we observe that the result for the deterministic code is weaker than that of the stochastic code. In the asymptotic regime, however, the restriction to only deterministic code is negligible since

$$
\frac{(\epsilon-\gamma) \beta^{-\frac{t}{1+t}} \log e}{n t \exp \left\{\frac{t}{1+t} G_{\frac{1}{1+t}}^{D, \epsilon}\left(X^{n}\right)\right\}} \rightarrow 0
$$

holds as $n \rightarrow \infty$.

\section{B. Theorem for a Prefix Code}

We have discussed a code without the prefix constraints. In this section, we discuss a result for an encoder $f^{\mathrm{p}}: \mathcal{X} \rightarrow$ $\{0,1\}^{\star}$ and a decoder $g^{\mathrm{p}}:\{0,1\}^{\star} \rightarrow \mathcal{Y}$ when we assume that $f^{\mathrm{p}}$ produces a prefix code.

As shown in (6), we have defined $R^{*}(D, \epsilon, t)$ for a nonprefix code. Similarly, we define $R_{\mathrm{p}}^{*}(D, \epsilon, t)$ as the fundamental limit on the normalized cumulant generating function of codeword lengths for a prefix code $\left(f^{\mathrm{p}}, g^{\mathrm{p}}\right)$. Then, a modification of the proof of Lemmas 1 and 2 yields the next result.

Theorem 6: For any $D \geq 0, \epsilon \in[0,1)$, and $t>0$,

$$
G_{\frac{1}{1+t}}^{D, \epsilon}(X) \leq R_{\mathrm{p}}^{*}(D, \epsilon, t) \leq G_{\frac{1}{1+t}}^{D, \epsilon}(X)+\left\lfloor\log k^{*}\right\rfloor+1,
$$

where $k^{*}$ is the integer satisfying (34) and (35).

\section{Proof: See Appendix B}

\section{Non-Asymptotics and Distortion Balls}

In our non-asymptotic analysis, the distortion $D$-ball around $y$ (i.e., (26) plays a crucial role. On the other hand, in the previous studies of non-asymptotics for lossy compression [3], [5], [6], [7], [8], the distortion $D$-ball around $x$ (i.e., $\tilde{\mathcal{B}}_{D}(x):=$ $\{y \in \mathcal{Y}: d(x, y) \leq D\})$ plays an important role. Investigating the relation of approaches between previous works and our work is one of the future works.

\footnotetext{
${ }^{6}$ Note that it holds that $\gamma \leq \epsilon$.
} 
APPENDIX A

Proof OF LEMMA 6

Define $\gamma$ as $\gamma=1-\sum_{i=1}^{k^{*}} \mathbb{P}\left[X \in \mathcal{A}_{D}\left(y_{i}\right)\right]$. Note that it holds that $\gamma \leq \epsilon$.

Now, we construct the following deterministic encoder $\hat{f}_{\text {det }}: \mathcal{X} \rightarrow\{0,1\}^{\star}$ and decoder $\hat{g}_{\text {det }}:\{0,1\}^{\star} \rightarrow \mathcal{Y}$.

\section{[Encoder]}

1) For $x \in \mathcal{A}_{D^{*}}\left(y_{i}\right)\left(i=1, \ldots, k^{*}\right)$, set $\hat{f}_{\text {det }}(x)=w_{i}$.

2) For $x \notin \bigcup_{i=1}^{k^{*}} \mathcal{A}_{D}\left(y_{i}\right)$, set $\hat{f}_{\text {det }}(x)=w_{1}$.

[Decoder] Set $\hat{g}_{\text {det }}\left(w_{i}\right)=y_{i}\left(i=1, \ldots, k^{*}\right)$.

First, we evaluate the excess distortion probability. From the definition of the encoder and the decoder,

$$
\begin{aligned}
\mathbb{P}\left[d\left(X, \hat{g}_{\text {det }}\left(\hat{f}_{\text {det }}(X)\right)\right) \leq D\right] & =\sum_{i=1}^{k^{*}} \mathbb{P}\left[X \in \mathcal{A}_{D}\left(y_{i}\right)\right] \\
& \geq 1-\epsilon .
\end{aligned}
$$

Therefore, we have

$$
\mathbb{P}\left[d\left(X, \hat{g}_{\text {det }}\left(\hat{f}_{\text {det }}(X)\right)\right)>D\right] \leq \epsilon
$$

Next, we evaluate the normalized cumulant generating function of codeword lengths for the code $\left(\hat{f}_{\text {det }}, \hat{g}_{\text {det }}\right)$. To this end, we denote by $\hat{Y}_{\text {det }}:=\hat{g}_{\text {det }}\left(\hat{f}_{\text {det }}(X)\right)$. For any $t>0$, we have

$$
\begin{aligned}
& \frac{1}{t} \log \mathbb{E}\left[2^{t \ell\left(\hat{g}_{\operatorname{det}}^{-1}\left(\hat{Y}_{\mathrm{det}}\right)\right)}\right] \\
& \stackrel{(a)}{\leq} \frac{1+t}{t} \log \sum_{j=1}^{k^{*}}\left[P_{\hat{Y}_{\mathrm{det}}}\left(y_{j}\right)\right]^{\frac{1}{1+t}} \\
& =\frac{1+t}{t} \log \left(\left[P_{\hat{Y}_{\mathrm{det}}}\left(y_{1}\right)\right]^{\frac{1}{1+t}}+\sum_{j=2}^{k^{*}-1}\left[P_{\hat{Y}_{\mathrm{det}}}\left(y_{j}\right)\right]^{\frac{1}{1+t}}\right. \\
& \left.+\left[P_{\hat{Y}_{\mathrm{det}}}\left(y_{k^{*}}\right)\right]^{\frac{1}{1+t}}\right) \\
& \leq \frac{1+t}{t} \log \left(\left[P_{\hat{Y}_{\mathrm{det}}}\left(y_{1}\right)+(\epsilon-\gamma)\right]^{\frac{1}{1+t}}\right. \\
& +\sum_{j=2}^{k^{*}-1}\left[P_{\hat{Y}_{\mathrm{det}}}\left(y_{j}\right)\right]^{\frac{1}{1+t}} \\
& \left.+\left[P_{\hat{Y}_{\mathrm{det}}}\left(y_{k^{*}}\right)-(\epsilon-\gamma)+(\epsilon-\gamma)\right]^{\frac{1}{1+t}}\right) \\
& \stackrel{(b)}{=} \frac{1+t}{t} \log \left(\left[P_{\hat{Y}}\left(y_{1}\right)\right]^{\frac{1}{1+t}}+\sum_{j=2}^{k^{*}-1}\left[P_{\hat{Y}}\left(y_{j}\right)\right]^{\frac{1}{1+t}}\right. \\
& \left.+\left[P_{\hat{Y}}\left(y_{k^{*}}\right)+(\epsilon-\gamma)\right]^{\frac{1}{1+t}}\right) \\
& \stackrel{(c)}{\leq} \frac{1+t}{t} \log \left(\sum_{j=1}^{k^{*}}\left[P_{\hat{Y}}\left(y_{j}\right)\right]^{\frac{1}{1+t}}+\frac{\epsilon-\gamma}{1+t}\left[P_{\hat{Y}}\left(y_{k^{*}}\right)\right]^{-\frac{t}{1+t}}\right) \\
& =\frac{1+t}{t} \log \left(\sum_{j=1}^{k^{*}}\left[P_{\hat{Y}}\left(y_{j}\right)\right]^{\frac{1}{1+t}}+\frac{\epsilon-\gamma}{1+t} \beta^{-\frac{t}{1+t}}\right)
\end{aligned}
$$

$$
\begin{aligned}
& \stackrel{(d)}{\leq} \frac{1+t}{t}\left\{\log \left(\sum_{j=1}^{k^{*}}\left[P_{\hat{Y}}\left(y_{j}\right)\right]^{\frac{1}{1+t}}\right)\right. \\
& \left.+\frac{(\epsilon-\gamma) \beta^{-\frac{t}{1+t}} \log e}{(1+t) \sum_{j=1}^{k^{*}}\left[P_{\hat{Y}}\left(y_{j}\right)\right]^{\frac{1}{1+t}}}\right\} \\
& =\frac{1+t}{t} \log \left(\sum_{j=1}^{k^{*}}\left[P_{\hat{Y}}\left(y_{j}\right)\right]^{\frac{1}{1+t}}\right)+\frac{(\epsilon-\gamma) \beta^{-\frac{t}{1+t}} \log e}{t \sum_{j=1}^{k^{*}}\left[P_{\hat{Y}}\left(y_{j}\right)\right]^{\frac{1}{1+t}}} \\
& \stackrel{(e)}{=} G_{\frac{1}{1+t}}^{D, \epsilon}(X)+\frac{(\epsilon-\gamma) \beta^{-\frac{t}{1+t}} \log e}{t \exp \left\{\frac{t}{1+t} G_{\frac{1}{1+t}}^{D, \epsilon}(X)\right\}}
\end{aligned}
$$

where $(a)$ follows from the same discussion as in (53), $(b)$ is due to the construction of $(\hat{f}, \hat{g})$ and $\left(\hat{f}_{\text {det }}, \hat{g}_{\text {det }}\right),(c)$ and $(d)$ follow from Taylor's expansion, and $(e)$ is due to 61).

Thus, we complete the proof of Lemma 6

\section{APPENDIX B}

\section{ProOf OF THEOREM 6}

We define a $(D, R, \epsilon, t)_{\mathrm{p}}$ code as follows.

Definition 10: Given $D, R \geq 0, \epsilon \in[0,1)$, and $t>0$, a prefix code $\left(f^{\mathrm{p}}, g^{\mathrm{p}}\right)$ satisfying

$$
\begin{aligned}
\mathbb{P}\left[d\left(X, g^{\mathrm{p}}\left(f^{\mathrm{p}}(X)\right)\right)>D\right] & \leq \epsilon, \\
\frac{1}{t} \log \mathbb{E}\left[2^{t \ell\left(f^{\mathrm{p}}(X)\right)}\right] & \leq R
\end{aligned}
$$

is called a $(D, R, \epsilon, t)_{\mathrm{p}}$ code.

Then, the fundamental limit that we investigate is

$$
R_{\mathrm{p}}^{*}(D, \epsilon, t):=\inf \left\{R: \exists \mathrm{a}(D, R, \epsilon, t)_{\mathrm{p}} \text { code }\right\} .
$$

To show Theorem 6, we prove the next two lemmas. If we prove these lemmas, we can immediately obtain Theorem 6 .

Lemma 7: For any $D \geq 0, \epsilon \in[0,1)$, and $t>0$, there exists a $(D, R, \epsilon, t)_{\mathrm{p}}$ code such that

$$
R=G_{\frac{1}{1+t}}^{D, \epsilon}(X)+\left\lfloor\log k^{*}\right\rfloor+1,
$$

where $k^{*}$ is the integer satisfying (34) and (35).

Lemma 8: For any $D \geq 0, \epsilon \in[0,1)$, and $t>0$, any $(D, R, \epsilon, t)_{\mathrm{p}}$ code satisfies

$$
R \geq G_{\frac{1}{1+t}}^{D, \epsilon}(X)
$$

Proof of Lemma 7. We use the same notations defined in Section VI-A. Further, we introduce the next notation: for $i=$ $1, \ldots, k^{*}$, a codeword $w_{i}^{\mathrm{p}}$ is defined as

$$
w_{i}^{\mathrm{p}}=w_{i} \circ h_{i},
$$

where $\circ$ denotes a concatenation and $h_{i} \in\{0,1\}^{\star}$ is a binary sequence such that $w_{i}^{\mathrm{p}} \neq w_{j}^{\mathrm{p}}(\forall i \neq j)$ and $\left\|h_{i}\right\|=\left\lfloor\log k^{*}\right\rfloor+$ $1-\lfloor\log i\rfloor$, where $\|\cdot\|$ denotes a length of a codeword. The definition of $w_{i}^{\mathrm{p}}$ indicates that the length of $w_{i}^{\mathrm{p}}$ is $\left\lfloor\log k^{*}\right\rfloor+1$ for all $i=1, \ldots, k^{*} 7$

\footnotetext{
${ }^{7}$ Note that the length of $w_{i}$ is $\lfloor\log i\rfloor$.
} 

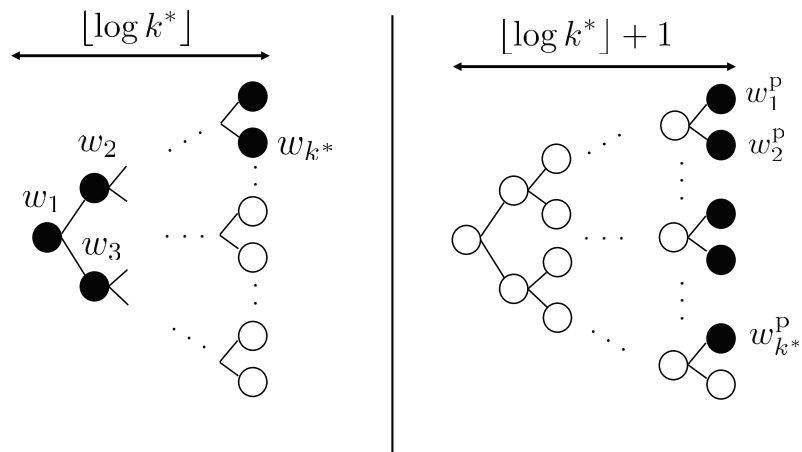

Fig. 1. Illustration of $w_{1}, w_{2}, \ldots, w_{k^{*}}$ and $w_{1}^{\mathrm{p}}, w_{2}^{\mathrm{p}}, \ldots, w_{k^{*}}^{\mathrm{p}}$ in a code tree.

Since the number of codewords $w_{1}, w_{2}, \ldots, w_{k^{*}}$ is at most

$$
1+2+2^{2}+\cdots+2^{\left\lfloor\log k^{*}\right\rfloor}=2^{\left\lfloor\log k^{*}\right\rfloor+1}-1,
$$

the codewords $w_{1}^{\mathrm{p}}, w_{2}^{\mathrm{p}}, \ldots, w_{k^{*}}^{\mathrm{p}}$ correspond to the leaf nodes of a code tree whose depth is $\left\lfloor\log k^{*}\right\rfloor+1$ (see Fig. 1). Thus, we can construct the following prefix code $\hat{f}^{\mathrm{p}}: \mathcal{X} \rightarrow\{0,1\}^{\star}$ and $\hat{g}^{\mathrm{p}}:\{0,1\}^{\star} \rightarrow \mathcal{Y}$.

\section{[Encoder]}

1) For $x \in \mathcal{A}_{D}\left(y_{i}\right)\left(i=1, \ldots, k^{*}-1\right)$, set $\hat{f}^{\mathrm{p}}(x)=w_{i}^{\mathrm{p}}$.

2) For $x \in \mathcal{A}_{D}\left(y_{k^{*}}\right)$, set

$$
\hat{f}^{\mathrm{p}}(x)= \begin{cases}w_{k^{*}}^{\mathrm{p}} & \text { with prob. } \frac{\beta}{\mathbb{P}\left[X \in \mathcal{A}_{D}\left(y_{k^{*}}\right)\right]}, \\ w_{1}^{\mathrm{p}} & \text { with prob. } 1-\frac{\beta}{\mathbb{P}\left[X \in \mathcal{A}_{D}\left(y_{k^{*}}\right)\right]} .\end{cases}
$$

3) For $x \notin \bigcup_{i=1}^{k^{*}} \mathcal{A}_{D}\left(y_{i}\right)$, set $\hat{f}^{\mathrm{p}}(x)=w_{1}^{\mathrm{p}}$.

[Decoder] Set $\hat{g}^{\mathrm{p}}\left(w_{i}\right)=y_{i}\left(i=1, \ldots, k^{*}\right)$.

Now, we evaluate the excess distortion probability of the code $\left(\hat{f}^{\mathrm{p}}, \hat{g}^{\mathrm{p}}\right)$. The same discussion as in the proof of Lemma 1 yields

$$
\mathbb{P}\left[d\left(X, \hat{g}^{\mathrm{p}}\left(\hat{f}^{\mathrm{p}}(X)\right)\right)>D\right]=\epsilon .
$$

Next, we evaluate the normalized cumulant generating function of codeword lengths for the code $\left(\hat{f}^{\mathrm{p}}, \hat{g}^{\mathrm{p}}\right)$ :

$$
\begin{aligned}
& \frac{1}{t} \log \mathbb{E}\left[2^{t \ell\left(\hat{f}^{p}(X)\right)}\right] \\
& \stackrel{(a)}{\leq} \frac{1}{t} \log \sum_{x \in \mathcal{X}} P_{X}(x) 2^{t \ell(\hat{f}(x))+t\left(\left\lfloor\log k^{*}\right\rfloor+1\right)} \\
& =\frac{1}{t} \log \left(\sum_{x \in \mathcal{X}} P_{X}(x) 2^{t \ell(\hat{f}(x))}\right)+\left\lfloor\log k^{*}\right\rfloor+1 \\
& =\frac{1}{t} \log \left(\mathbb{E}\left[2^{t \ell(\hat{f}(X))}\right]\right)+\left\lfloor\log k^{*}\right\rfloor+1 \\
& \stackrel{(b)}{\leq} G_{\frac{1}{1+t}}^{D, \epsilon}(X)+\left\lfloor\log k^{*}\right\rfloor+1
\end{aligned}
$$

where $(a)$ follows from the construction of $(\hat{f}, \hat{g})$ in Section VI-A and that of $\left(\hat{f}^{\mathrm{p}}, \hat{g}^{\mathrm{p}}\right)$, and $(b)$ is due to 62. This completes the proof of Lemma 7

\section{Proof of Lemma 8 .}

By replacing (65) with Kraft's inequality and following the same route as in the proof of Lemma 2, we obtain Lemma 8 .

\section{ACKNOWLEDGMENT}

The authors would like to thank Dr. Hideki Yagi for helpful discussions. This work was supported in part by JSPS KAKENHI Grant Numbers JP16K00195, JP16K00417, JP17K00316, JP17K06446, and by Waseda University Grant for Special Research Projects (Project number: 2017A-022).

\section{REFERENCES}

[1] L. L. Campbell, "A coding theorem and Rényi's entropy," Information and Control, vol. 8, no. 4, pp. 423-429, 1965.

[2] T. A. Courtade and S. Verdú, "Cumulant generating function of codeword lengths in optimal lossless compression," Proc. IEEE Int. Symp. Inf. Theory (ISIT), Honolulu, HI, USA, pp. 2494-2498, June-July 2014.

[3] T. A. Courtade and S. Verdú, "Variable-length lossy compression and channel coding: non-asymptotic converses via cumulant generating functions," Proc. IEEE Int. Symp. Inf. Theory (ISIT), Honolulu, HI, USA, pp. 2499-2503, June-July 2014.

[4] A. Feinstein, "A new basic theorem of information theory," IRE Trans. Inf. Theory, vol. 4, pp. 2-22, Sept. 1954.

[5] I. Kontoyiannis, "Pointwise redundancy in lossy data compression and universal lossy data compression," IEEE Trans. Inf. Theory, vol. 46, no. 1, pp. 136-152, Jan. 2000.

[6] I. Kontoyiannis and J. Zhang, "Arbitrary source models and Bayesian codebooks in rate-distortion theory,'IEEE Trans. Inf. Theory, vol. 48, no. 8, pp. 2276-2290, Aug. 2002.

[7] V. Kostina and S. Verdú, "Fixed-length lossy compression in the finite blocklength regime," IEEE Trans. Inf. Theory, vol. 58, no. 6, pp. 33093338, June 2012.

[8] V. Kostina, Y. Polyanskiy, and S. Verdú, "Variable-length compression allowing errors," IEEE Trans. Inf. Theory, vol. 61, no. 8, pp. 4316-4330, Aug. 2015.

[9] S. Kuzuoka, "On the smooth Rényi entropy and variable-length source coding allowing errors," Proc. IEEE Int. Symp. Inf. Theory (ISIT), Barcelona, Spain, pp. 745-749, July 2016.

[10] A. W. Marshall, I. Olkin, and B. C. Arnold, Inequalities: Theory of Majorization and Its Applications, Second Edition, Springer, 2010.

[11] E. C. Posner, E. R. Rodemich, and H. Rumsey, "Epsilon entropy of stochastic processes," Ann. Math. Statist., vol. 38, no. 4, pp. 1000-1020, Aug. 1967.

[12] A. Rényi, "On measures of entropy and information," 4th Berkley Symposium on Mathematics, Statistics and Probability, pp. 547-561, 1960.

[13] S. Saito, H. Yagi, and T. Matsushima, "Variable-length lossy compression allowing positive overflow and excess distortion probabilities," Proc. IEEE Int. Symp. Inf. Theory (ISIT), Aachen, Germany, pp. 15681572, June 2017.

[14] S. Saito and T. Matsushima, "Cumulant generating function of codeword lengths in variable-length lossy compression allowing positive excess distortion probability," [Online]. Available: http://arxiv.org/abs/1801.02496 